\title{
Shock Reliability Enhancement for MEMS Vibration Energy Harvesters with Nonlinear Air Damping as Soft Stopper
}

\author{
$\underline{\text { Shao-Tuan Chen }}{ }^{1}$, Sijun Du ${ }^{1}$, Emmanuelle Arroyo ${ }^{1}, \mathrm{Yu} \mathrm{Jia}^{1,2}$ \\ and Ashwin Seshia ${ }^{1}$ \\ ${ }^{1}$ Nanoscience Centre, University of Cambridge, Cambridge, CB3 OFF, UK \\ ${ }^{2}$ Department of Mechanical Engineering, University of Chester, Chester CH1 4BJ, UK \\ E-mail: stc33@cam.ac.uk
}

Abstract. This paper presents a novel application of utilising nonlinear air damping as soft mechanical stopper to increase the shock reliability for MEMS vibration energy harvesters. Theoretical framework for nonlinear air damping is constructed for MEMS vibration energy harvesters operating in different air pressure levels, and characterisation experiments are conducted to establish the relationship between air pressure and nonlinear air damping coefficient for rectangular cantilever MEMS micro cantilevers with different proof masses. Design guidelines on choosing the optimal air pressure level for different MEMS vibration energy harvesters based on the trade-off between harvestable energy and the device robustness is presented, and random excitation experiments are performed to verify the robustness of MEMS vibration energy harvesters with nonlinear air damping as soft stoppers to limit the maximum deflection distance and increase the shock reliability of the device.

\section{Introduction}

With the development of wireless electronic devices, micro-scale regenerative power sources have attracted increasing research interests in recent years, as they offer the potential of wireless and self-sufficient powering without the burden of expensive intervention maintenance or replacement compared to their battery counterparts.

Miniaturised energy harvesters have been developed with Microelectromechanical systems (MEMS) technology, as it promises the possibility for close in-package [1] or monolithic cointegration with conventional semiconductor technology [2].

Of all the common alternative energy sources, including solar energy [3], thermal energy [4] and mechanical energy [5], vibration-driven piezoelectric vibration energy harvester (PVEH), 
which transforms ambient kinetic energy into electric power using piezoelectric materials has surfaced as an attractive approach, as the source of vibrational mechanical energy is ubiquitous, such as in moving machineries or many transportation-related built infrastructure.

Despite the potential of MEMS Piezoelectric Vibration Energy Harvesters (PVEHs), commercialisation and real-world application for such technology is still limited, mainly due to the lack of robustness of MEMS devices. The dominant material used for MEMS manufacture is silicon (in single crystal or polycrystalline forms), which is particularly prone to fracture caused by excessive stress resulting from large amplitude vibration or instantaneous shocks and dynamic excitations under variable environmental conditions. An example of device fracture under such loading conditions is shown in Fig.1.

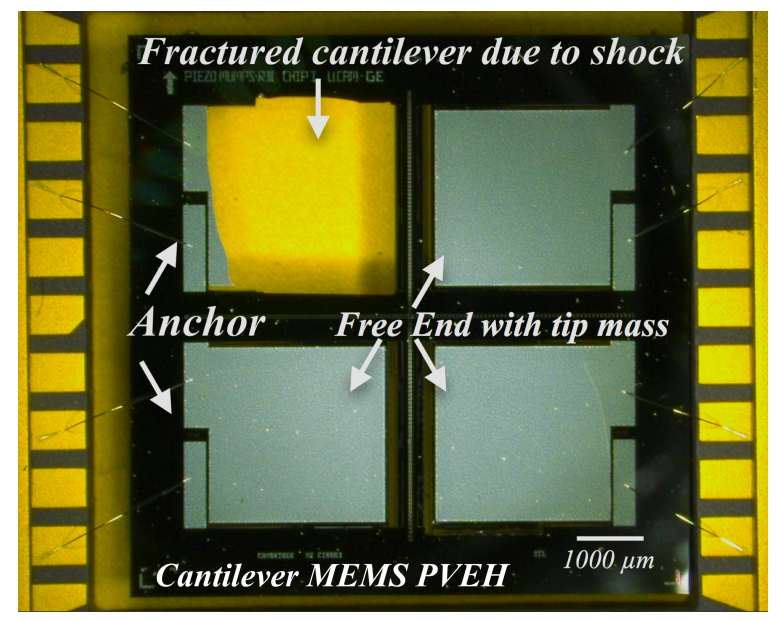

Figure 1. Picture of MEMS PVEH [6] The top left corner reveals fractured cantilever due to excessive shock loading.

Previous researchers have utilised mechanical stoppers to restrict the maximum displacement of the MEMS PVEH to improve the shock reliability for harsh environment deployment. Renaud et al. [7] designed a package for MEMS PVEH with steps which can act as a protective stopper for the PVEH by restricting the maximum deflection distance of the stopper. The reduced gap between the chip carrier and the PVEH is designed to be located at the edge of the proof mass of the cantilever MEMS vibration energy harvesting devices, with aim to reduce the deformation and the strain at the anchor of the device. The survival rate of the devices increased from $4 \%$ to $60 \%$ before and after the implementation of the step stopper. However, as the collision between the stopper and the devices is elastic, the forces exerted on the device when the stopper is engaged is still large enough to cause chipping damage on the device.

Renaud et al. [8] later devised a stopper for MEMS PVEH by the utilisation of spring anchors to redirect the impact force from the anchor of the device where it is most fragile to other less fragile parts of the PVEH. They designed three types of shock absorbing structures, namely rigid stoppers, Parylene-covered stoppers and silicon-only flexible stoppers. For rigid and flexible stoppers, the improvement in terms of shock reliability is limited. Parylene-covered stoppers resulted in significant improvement in terms of shock reliability, however the integration of Parylene is an additional fabrication step and introducing process complexity.

These studies have proven that the shock reliability of the MEMS PVEH can be enhanced by restricting the maximum displacement of the device with stoppers. However, the impact when 
the MEMS PVEH engages with the mechanical stopper can damage the MEMS PVEH, rendering the MEMS PVEH with mechanical stoppers still suspect to fracture due to excessive dynamic loading.

As an alternative, nonlinear air damping has previously been observed by Jia et al. [6] to serve as a potential candidate as soft mechanical stopper for MEMS PVEHs. While vacuum packaging is typically used in MEMS applications to minimise air damping and improve the overall quality factor and performance, the lack of damping mechanisms for vacuum packaged PVEHs often result in fragile devices when deployed in environments with variable excitation conditions. Chen et al. [9] also observed that for higher excitation amplitude, the maximum displacement of the PVEH is greatly diminished when the PVEH is operating in room air pressure compared to vacuum condition. Amplitude saturation occurs under high air pressure levels when the excitation amplitude is high, as the maximum displacement changes slightly when the excitation amplitude is further increased. As for lower excitation amplitudes, the maximum displacement of the PVEH is less affected by the ambient air pressure level. This phenomenon caused by nonlinear air damping is suitable for the utilisation as stopper, as the nonlinear air damping does not influence the dynamic behaviour of the PVEH when the device is operating in low and mild excitation scenarios, and hinders the displacement level when the excitation level is high and excessive, thus protecting the device from fracturing due to excessive stress when subjected to shocks or excessive loadings. Instead of impacting the device when the stopper is engaged, soft mechanical stoppers with nonlinear air damping acts as additional form of energy dissipation mechanism, thus eliminating the probability of damaging the device when the stopper is engaged.

This paper reports on the theory and experimental verification on utilising air damping as a soft stopper mechanism for piezoelectric vibration energy harvester (PVEH) to enhance shock resistance ability for PVEH. The PVEH utilised in this study is designed by Jia et al. [6] as a cantilever with proof mass at the free end. Theoretical framework for nonlinear air damping is introduced to identify the origin of nonlinear damping for MEMS PVEH operating in air, and the relationship between air pressure and the power output of the MEMS PVEH is obtained. Characterisation experiments are conducted to relate the nonlinear damping coefficient with the ambient air pressure and the geometry of the MEMS PVEH. Lastly, application experiments are performed under different air pressure levels to demonstrate the effect of squeeze film damping on the system response, and the power output of devices with different air pressure levels under random excitations.

\section{Modelling}

The dynamic behaviour of a MEMS PVEH has been represented as a linear spring-massdamper system by Kaźmierski et al. [10], as shown in the schematic in Fig. 2. The system is comprised of an inertial mass $m$, a spring with stiffness $k$ and a damping coefficient $c$. The equation of motion for such devices can be modelled as a linear second order spring mass resonant system with a linear energy dissipation damping term, which accounts for the combination of mechanical parasitic losses and the conversion loss of energy transfer from the mechanical to electrical domains. The assumption that the electromechanical coupling can be modelled as an additional viscous damping term has been established and employed in several previous works [11]. 

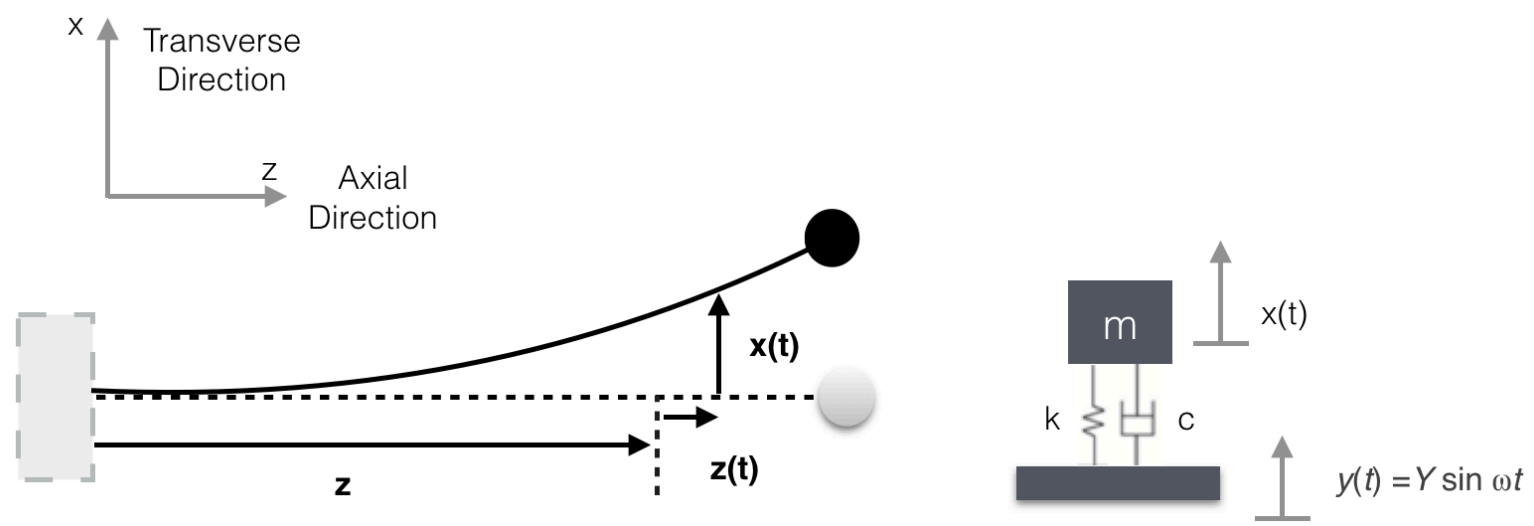

Figure 2. On the left is the schematic of a MEMS microcantilever with tip mass. The transverse displacement is denoted as $x(t)$ and the axial displacement is $z(t)$, on the right is the equivalent model of Spring-Mass-Damper System depicting the MEMS microcantilever

However, in real-world applications where complex couplings exist between the devices and external environments, the energy dissipation mechanisms for MEMS PVEHs have shown to be a combination of both linear and nonlinear effects [12]. Therefore, in addition to the linear damping mechanisms, nonlinear damping must be considered when modelling the dynamic behaviour of MEMS PVEHs. The nonlinear energy dissipation might originate from electromechanical piezoelectric coupling [13], friction, such as Coulomb friction [14] or aerodynamic drag friction [15], which the damping forces were found to be proportional to the squared of velocity of the system, instead of linearly proportional to the velocity of the system as in viscous damping.

\subsection{Piezoelectric coupling for PVEH}

For a nonlinear MEMS piezoelectric vibration energy harvester with nonlinear linear damping forces, the general equation of motion and the corresponding current equation of the harvester becomes:

$$
\begin{gathered}
m x \ddot{(t})+C(x) \dot{x}(t)+K(x) x(t)+\theta V_{p}(t)=y(t) \\
\theta \dot{x}(t)-C_{p} V_{p}(t)=\frac{V_{p}(t)}{R}(2)
\end{gathered}
$$

where $m$ is the effective mass of the microcantilever, $C$ comprises of a polynomial term representing the nonlinear dissipative mechanical damping, $K$ is the stiffness of the structure, $x$ is relative the displacement of the beam tip, $y(t)$ represents the external excitation, $\theta$ is the piezoelectric constant (forcing factor) which is the electromechanical coupling coefficient for MEMS piezoelectric vibration energy harvester, $V_{p}(t)$ is the terminal voltage of the vibration energy harvester, $R$ is load resistance the piezoelectric vibration energy harvester, and $C_{p}$ is the intrinsic capacitance of the MEMS PVEH.

Eqn.1 is the mechanical governing equation of the MEMS PVEH including the electromechanical coupling term, and Eqn.2 is the constitutive relation for the electrical domain of the MEMS PVEH. For modelling the dynamic behaviour of PVEHs, two equations need to be solved simultaneously to obtain the relationship between the output voltage of the piezoelectric energy harvester and the displacement of the MEMS cantilever.

Roundy et al. [16] first proposed the single degree-of-freedom models for the piezoelectric vibration energy harvesters, which incorporate the electrical domain and dynamic domain. Lu 
et al. [17] later developed the distributed parameters model, however the electromechanical coupling was neglected in the computation of displacement in the mechanical domain. This leads to inaccurate prediction of the mechanical response as well as the electrical power output. Chen et al. [18] combined the mechanical and electric domain for the electromechanical coupling model, where the piezoelectric coupling was modelled as a simple viscous damping term. However, Erturk et al. [19] demonstrates the viscous damping model for the piezoelectric coupling effect is an oversimplification for the distributed parameter modelling, and Erturk et al. [20] later demonstrated that the piezoelectric coupling between the electrical and mechanical domain affects both the damping ratio of the dynamic system and the natural frequency of the system under harmonic base excitation condition. As a result, Tan et al. [21] developed a decoupled model which links the analytical relation between the displacement of the MEMS cantilever in the mechanical domain and the power output of the piezoelectric vibration energy harvester in the electrical domain into a single equation. The approach Tan et al. took assumes that the dynamic as well as the electric constitutive equation both have the solution of the harmonic form as:

$$
x(t)=\frac{1}{2} a_{1} e^{i \Omega t}+C_{1}
$$

and

$$
V(t)=\frac{1}{2} a_{2} e^{i \Omega t}+C_{2}
$$

where $a_{1}$ and $a_{2}$ are the mode coordinate and voltage of the constitutive equation, $\Omega$ is the frequency of the mode coordinate, while $C_{1}$ and $C_{2}$ are the complex conjugates of the solution.

Tan et al. found the relationship between Eqn.3 and Eqn.2 by expressing the voltage output from the electric domain as a function of the amplitude of the mode coordinate from linking the two mode coordinates as:

$$
a_{2}=\frac{R \theta_{p} \Omega}{\sqrt{C_{p}^{2} \Omega^{2} R^{2}+1}}\left|a_{1}\right|
$$

and the maximum voltage power output is then expressed as:

$$
V=\frac{R \theta_{p} \Omega}{\sqrt{C_{p}^{2} \Omega^{2} R^{2}+1}}\left|a_{1}\right|
$$

Finally, the piezoelectric coupling term in Eqn. 1 can be expressed as a function of $a_{1}, x(t)$ and $x(t)$ as:

$$
\theta_{p} V(t)=\frac{R^{2} \theta_{p}^{2} \Omega^{2} C_{p} x(t)+R \theta_{p}^{2} x(t)}{C_{p}^{2} \Omega^{2} R^{2}+1}
$$

and the decoupled equation for the dynamic equation, which incorporates the piezoelectric coupling into the mechanical domain is written as:

$$
x \ddot{(t})+2 \bar{\zeta} \overline{\omega_{n}} x \dot{(t)}+{\overline{\omega_{n}}}^{2} x(t)=y(t)(8)
$$

where $\overline{\omega_{n}}$ and $\bar{\zeta}$ are the modified natural frequency of the system and the modified damping ratio respectively, which is given by: 


$$
\left.\overline{\omega_{n}}=\sqrt{\omega_{n}^{2}+\frac{R^{2} \theta_{p}^{2} \Omega^{2} C_{p}}{R^{2} C_{p}^{2} \Omega^{2}+1}}, \bar{\zeta}=\frac{\left(2 \zeta \omega_{n}+\frac{R \theta p^{2}}{C_{p}^{2} \Omega^{2} R^{2}+1}\right.}{2 \overline{\omega_{n}}}\right)
$$

\subsection{Nonlinear air damping for PVEH}

One of the problems of considering complex and nonlinear damping such as dry friction, air drag damping and squeeze film effect lies in the fact that these types of nonlinear energy dissipation mechanisms give rise to nonlinear terms in the governing differential equation of the system, whereas the exact mathematical solutions for nonlinear differential equations are unobtainable even for simple geometries and boundary conditions. Instead, the system dynamics and the values of nonlinear parameters are usually determined empirically.

Ravindra [22] has identified the representation of damping force in the equation of motion for soft Duffing oscillators with nonlinear damping. The nonlinear damping force in this case can be represented as:

$$
\zeta_{n} \dot{x}|\dot{x}|^{p-1}(10)
$$

Where $p$ is the nonlinear damping exponent, and $\zeta_{n}$ is the corresponding nonlinear damping coefficient in the equation of motion. The precise value of the damping exponent $p$ can be determined both experimentally and theoretically by considering the origin of the damping for the given system. For MEMS PVEHs operating in air, the nonlinear damping is caused by the interaction between the MEMS devices and the surrounding fluid medium, therefore, fluid drag damping and squeeze film damping should be considered when modelling the dynamic behaviour of such MEMS devices. For fluid drag damping, the drag force caused the vibration of the device is found to be proportional to the velocity squared of the vibrating velocity of the device [23].

Moreover, Hosaka et al. [24] have formulated the energy dissipation mechanism of vibrating MEMS cantilevers due to air drag and squeeze film damping, with the formulations summarised as below. Fig. 3 depicts the schematic of the damping model for a cantilever vibrating in fluid with operative nonlinear damping mechanisms shown.

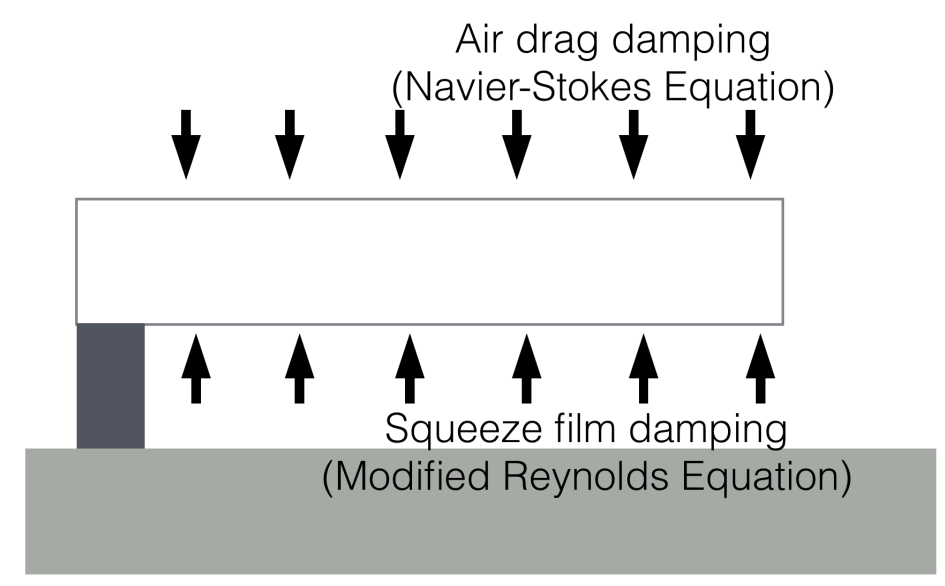

Figure 3. Energy dissipation mechanisms for microcantilever vibrating in fluid with a combination of squeeze film damping and air drag damping. 
The damping term due to fluid drag is obtained by approximating the micro-cantilever as a continuous beam model. The dynamic Euler-Bernoulli beam equation of such system when subjected to external excitation with excitation frequency $\omega$ and nonlinear damping due to fluid drag is given as [17]:

$$
\rho_{b} b h \frac{\partial^{2} w}{\partial t^{2}}+\frac{\beta}{b}\left(\frac{\partial w}{\partial t^{2}}\right)^{2}+E I \frac{\partial^{4} w}{\partial t^{4}}=f e^{i \omega t}
$$

Where

$$
\beta=3 \pi \mu b+\frac{3}{4} \pi b^{2} \sqrt{2 \rho_{a} \mu \omega}
$$

which is related to the damping force created by the fluid flow pass through individual spheres. The width and thickness of the rectangular cantilever is denoted as $b, h$, while $\rho_{a}$ and $\rho_{b}$ represents the density of the silicon substrate and the fluid medium respectively. $\mu$ denotes the viscosity of the fluid. $w, I, x$ and $f$ represents the deflection at the longitudinal position, moment of inertia of the beam, the longitudinal position along the cantilever and the amplitude of the external excitation.

The deflection $w$ of the beam can be decomposed by utilising the normal mode function $\phi_{n}$, and the time function $w_{n}$ as in Eqn. 13 as:

$$
\begin{gathered}
w(x, t)=\sum_{n=1}^{\infty} w_{n}(t) \cdot \phi_{n}(x) \\
m_{n} \ddot{w}_{n}+c_{n} \dot{w}_{n}^{2}+k_{n} w_{n}=f_{n} e^{i \omega t}
\end{gathered}
$$

where

$$
\begin{gathered}
m_{n}=\rho_{b} b h \int_{0}^{L} \phi_{n}^{2} d x, f_{n}=\int_{0}^{L} f \phi_{n} d x \\
c_{1 n}=\frac{\beta m_{n}}{{ }^{b^{2} h}}, k_{n}=\omega_{n}^{2} m_{n}(16)
\end{gathered}
$$

When the cantilever is oscillating by the $n$th resonant frequency $\omega_{n}$, the damping ratio $\zeta_{1 n}$ is then obtained as [24]:

$$
\zeta_{1 n}=\frac{\bar{c}_{1 n}}{2 m_{n} \omega_{n}}=\frac{\bar{\beta}}{2 \rho_{b} h b^{2} \omega_{n}}(17)
$$

where $\zeta_{\text {In }}$ denotes the nonlinear damping ratio contributed by the fluid drag. For a cantilever with proof mass at the free vibrating end, the damping ratio for air drag can be further divided to $\zeta_{\text {In, beam }}$ and $\zeta_{\text {In,mass }}$ as [25]:

$$
\begin{aligned}
\zeta_{1 \text {, beam }} & =\frac{1}{b} \frac{\bar{\beta}}{2 m_{\text {beam }} \omega_{n}} \\
\zeta_{1, \text { mass }} & =\frac{\bar{\beta}}{2 m_{\text {mass }} \omega_{n}}
\end{aligned}
$$

As for the nonlinear squeeze film damping, the governing equation motion is given by the modified Reynolds Equation as: 


$$
\frac{\partial^{2} p}{\partial x^{2}}=\frac{12}{g_{0}^{3}} \frac{\partial w}{\partial t}(20)
$$

where $p$ is the air pressure in the thin-film region between the cantilever and the bottom substrate and $g_{0}$ is the original thickness of the gap. The air pressure $p$ can be calculated by integrating Eqn. 20 with a boundary condition of $p=0$ at $\mathrm{y}= \pm 0.5 \mathrm{~b}$. Similar to the air drag damping formulation, the dynamic Euler-Bernoulli beam equation of such system when subjected to external excitation with excitation frequency $\omega$ and nonlinear damping due to squeeze film damping is given as:

$$
\rho_{b} b h \frac{\partial^{2} w}{\partial t^{2}}+\frac{\mu b^{3}}{g_{0}^{3}}\left(\frac{\partial w}{\partial t^{2}}\right)^{2}+E I \frac{\partial^{4} w}{\partial t^{4}}=f e^{i \omega t}
$$

And the damping ratio for squeeze film damping can be obtained by decomposition as:

$$
\zeta_{2 n}=\frac{\mu b^{2}}{2 \rho_{b} h g_{0}{ }^{3} \omega_{n}}(22)
$$

As a result, the nonlinear damping coefficient $\zeta_{n}$ in the equation of motion for cantilever vibrating in a fluid medium can be a combination of both air drag and squeeze film damping as:

$$
\zeta_{n}=\zeta_{1 n}+\zeta_{2 n}=\frac{1}{b} \frac{3 \pi \mu b+\frac{3}{4} \pi b^{2} \sqrt{2 \rho_{a} \mu \omega}}{2 m_{\text {beam }} \omega_{n}}+\frac{3 \pi \mu b+\frac{3}{4} \pi b^{2} \sqrt{2 \rho_{a} \mu \omega}}{2 \rho_{b} 2 m_{\text {mass }} \omega_{n}}+\frac{\mu b^{2}}{2 \rho_{b} h g_{0}{ }^{3} \omega_{n}}
$$

which shows the damping coefficient is a function of the dimensions of the cantilever, the density of the fluid medium and the initial gap between the microcantilever and the substrate.

The equation of motion for a MEMS PVEH operating in air can be depicted by a Duffing equation considering the piezoelectric coupling effect and a nonlinear damping term described above as:

$$
\ddot{x}+2 \bar{\zeta} \overline{\omega_{n}} \dot{x}+\zeta_{n} \dot{x}|\dot{x}|+\mu x^{3}+{\overline{\omega_{n}}}^{2} x=A \omega^{2} \cos (\omega t)
$$

where $x$ is the displacement of the MEMS PVEH, $\bar{\zeta}$ and $\zeta_{n}$ is modified linear damping ratio and the nonlinear damping representing the combination of nonlinear air drag and squeeze damping respectively, $\mu$ is the Duffing coefficient representing the nonlinear stiffness of the system, $\bar{\omega}_{n}$ is the modified natural frequency of the MEMS PVEH, $A$ is the amplitude of the external excitation, $\omega$ is the excitation frequency and $t$ is the time domain.

Fig. 4 depicts the maximum displacement of the model in Eqn. 24 when subjected to external excitation with different amplitude. The parameters used in this dimensionless model are chosen as $\bar{\zeta}=8.16 \times 10^{-4}, \overline{\omega_{n}}=1237 \mathrm{rad} / \mathrm{s}$ and $\mu=3 \times 10^{-4}\left(\mathrm{~ms}^{-2}\right.$, where parameters used in this schematic are based on the measurement of the MEMS vibration energy harvester, where the piezoelectric coupling coefficient is defined as $\theta_{p}{ }^{2}=\frac{d_{31}{ }^{2}}{\varepsilon_{33} s_{11}}$ [26], where $d_{31}$ is the piezoelectric strain constant, $s_{11}$ is the elastic compliance, and $\varepsilon_{33}$ is the permittivity under constant stress of the piezoelectric material. As a result, the piezoelectric coupling coefficient $\theta_{p}=0.25$ for the piezoelectric material being Aluminium Nitride. The capacitance of the 
MEMS vibration harvester $C_{p}=0.08 \mathrm{nF}$, and the load resistance of the MEMS vibration harvester is $300 \mathrm{k} \Omega$. As seen from the graph, the maximum displacement of the system can be changed by changing the nonlinear air damping coefficient. For system with lower nonlinear damping coefficient, the relationship between the maximum displacement and the excitation amplitude is more linear, while the maximum displacement saturates for higher excitation amplitude when the nonlinear damping coefficient is high.

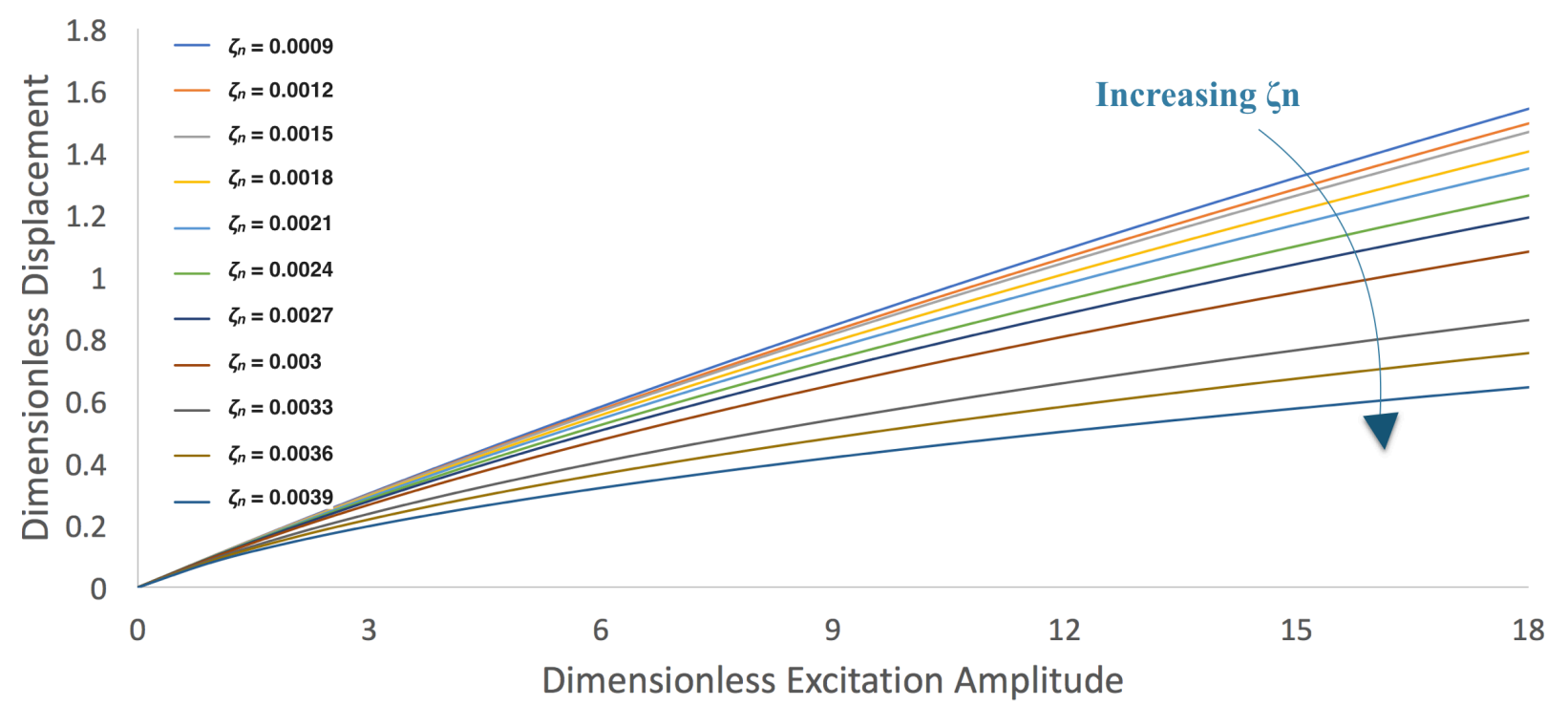

Figure 4. Dimensionless model demonstrating the dynamic response of MEMS PVEH with different values of nonlinear air damping $\zeta_{n}$.

\section{Experimental characterisation}

\subsection{Device fabrication}

The MEMS Piezoelectric Vibration Energy Harvester (PVEH) utilised in this study is of rectangular cantilever topology, which represents the most popular geometrical design for inertial vibration energy harvesters. The PVEH is designed by Jia et al. [6] as a rectangular cantilever with proof mass suspended at the free moving end and clamped at the other end. The dimensions of the cantilever are $3.5 \mathrm{~mm}$ by $3.5 \mathrm{~mm}$, consisting of Aluminium Nitride (AIN) piezoelectric layer with thickness of $0.5 \mu \mathrm{m}$ and $10 \mu \mathrm{m}$ of doped Si process. The proof mass of the cantilever is comprised of $400 \mu \mathrm{m}$ of un-etched silicon substrate. Four devices with different proof masses were utilised in this study, and are distinguished by the proof-mass-tocantilever-length ratio between each device. The specific mass-to-length ratio are $40 \%, 50 \%$, $60 \%$ and $70 \%$, and the devices were named as MC40, MC50, MC60 and MC70, respectively. The PVEH is then covered with an electrode layer consisting of $1.02 \mu \mathrm{m}$ of Aluminium. Fig. 5 shows a single MEMS chip consisting of MC40, MC50, MC60 and MC70 mounted in a chip carrier. 


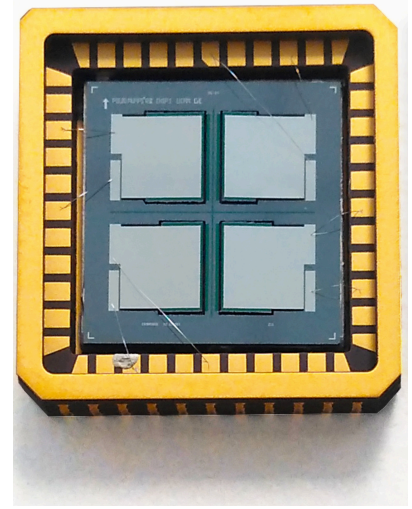

Figure 5. Photograph of the MEMS chip consisting four cantilever vibration energy harvesters with different proof mass

\subsection{Experimental setup}

The experimental setup with the assembled device of the MEMS PVEH is depicted in Fig.6. The silicon die of the cantilevers is attached to a chip carrier with a square spacer, which is utilised to accommodate the large displacement of the cantilever when the PVEH is excited by external vibration. The PVEH is mounted to an electromagnetic shaker (LDS V406 M4-CE) with a custom PCB. A function generator (Agilent Technologies 33250A $80 \mathrm{MHz}$ waveform generator) is utilised to provide electrical excitation to drive the shaker. The entire PVEH including the shaker is then placed into a vacuum chamber, where the pressure can be controlled and monitored throughout the experiments. Characterisation experiments are performed by varying the amplitude of the sinusoidal external excitation from 0.5 to $7 \mathrm{~m} / \mathrm{s}^{2}$ and air pressure inside the vacuum chamber from $1 \mathrm{mbar}, 100 \mathrm{mbar}, 200 \mathrm{mbar}, 300 \mathrm{mbar}, 500 \mathrm{mbar}$, 800 mbar to 1000 mbar, respectively. The output RMS voltage of the PVEH is recorded while the device is connected to an optimal resistive load of $300 \mathrm{k} \Omega$.
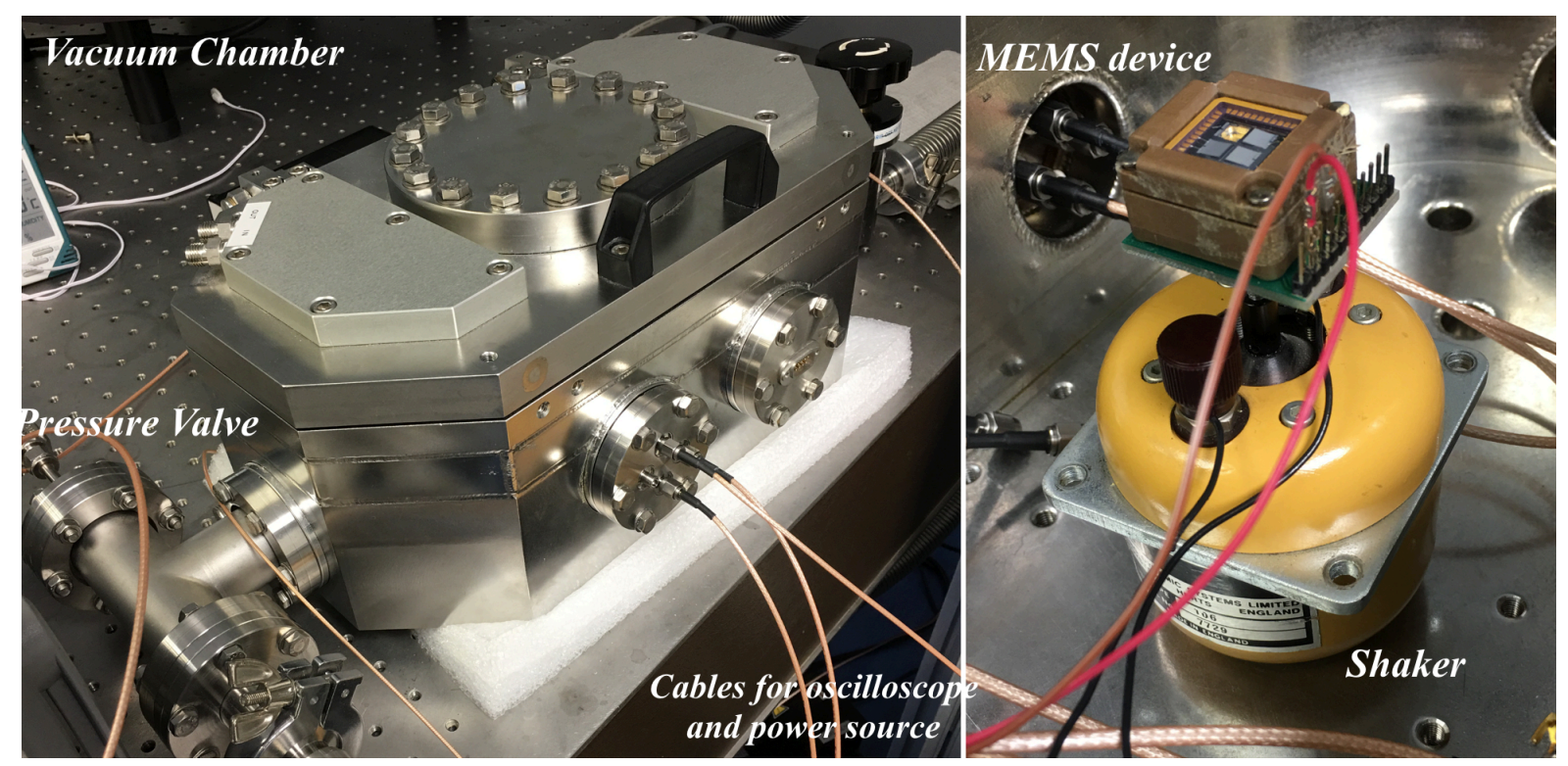

Figure 6. Experimental setup, characterisation experiments are performed when the MEMS PVEH is mounted to a shaker and put into a vacuum chamber to adjust the ambient air pressure. 
The power generated of each device at their respective resonant frequency when ambient air pressure is at room pressure is presented in Table 1 . As seen from the values recorded in the table, the resonant frequency of the cantilever increases as the proof mass ratio increases from MC40 to MC70, also the maximum power generated increases from MC40 to MC70 owing to the larger proof mass and larger displacement when subjected to the same excitation amplitude under their respective resonant frequency.

Table 1. Peak power output and normalised power density driving at $0.35 \mathrm{~g}$ when ambient air pressure is at room pressure

\begin{tabular}{|c|c|c|c|c|c|}
\hline Device & $\begin{array}{c}\text { Voltage } \\
\mathrm{V}\end{array}$ & $\begin{array}{c}\text { Vibration g } \\
(0-\text {-Peak })\end{array}$ & $\begin{array}{c}\text { Natural } \\
\text { Frequency }(\mathrm{Hz})\end{array}$ & $\begin{array}{c}\text { Peak Power } \\
\text { Output }(\mu \mathrm{W})\end{array}$ & $\begin{array}{c}\text { Normalised Power } \\
\text { Density }\left(\mu \mathrm{W} / \mathrm{cm}^{3} /(\mathrm{m} / \mathrm{s})^{2}\right)\end{array}$ \\
\hline MC70 & 1.13 & 0.35 & 215.0 & 4.32 & 155 \\
\hline MC60 & 1.02 & 0.35 & 202.2 & 3.48 & 77 \\
\hline MC50 & 0.96 & 0.35 & 199.9 & 3.12 & 58 \\
\hline MC40 & 0.81 & 0.35 & 194.5 & 2.13 & 40 \\
\hline
\end{tabular}

Fig. 7 shows the maximum power output of each device as a function of input excitation amplitude when the devices are driven at the respective resonant frequencies with sinusoidal excitation at 1 mbar.

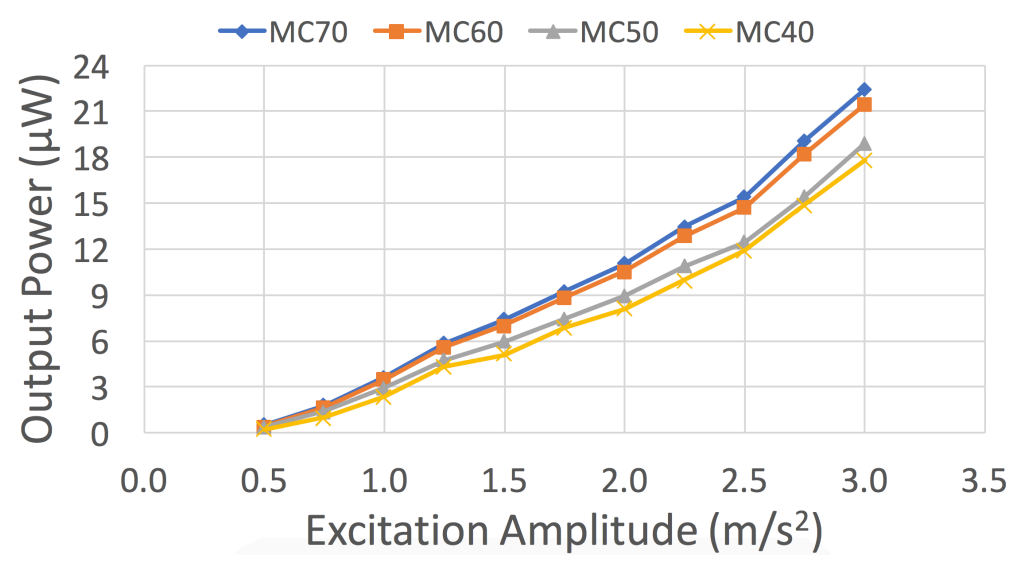

Figure 7. Power curve for 4 tested devices driving at resonant frequency at $p=1$ mbar

As seen from the graph, the power output of the MEMS PVEHs increases as the ratio of the proof mass increases, as the maximum power output reaches $22.5 \mu \mathrm{W}, 21.4 \mu \mathrm{W}, 18.9 \mu \mathrm{W}$ and $17.8 \mu \mathrm{W}$ for MC70, MC60, MC50 and MC40 respectively when the external excitation amplitude is at around $3 \mathrm{~m} / \mathrm{s}^{2}$. The normalised power density of each device operating under ambient pressure of 1 mbar is recorded in Table. 2 and compared against other MEMS PVEHs operated in vacuum conditions in the literature. Although the normalised power density of all four devices are comparable to other reported devices tested in vacuum condition, all tested devices fracture soon after the excitation amplitude reach beyond the reported values on Fig. 8 , making this configuration of near vacuum for MEMS PVEHs not suitable to be deployed in dynamic scenarios where shocks or large amplitude vibrations might occur. 
Table 2. Peak power output of the four tested devices driven at $P=1 \mathrm{mbar}$, and comparison with other vacuum packaged vibration energy harvesters in the literature

\begin{tabular}{|c|c|c|c|c|c|}
\hline Reference & $\begin{array}{c}\text { Volume } \\
\mathrm{mm}^{3}\end{array}$ & $\begin{array}{c}\text { Input vibration } \\
\mathrm{g}(0-\text { Peak })\end{array}$ & $\begin{array}{c}\text { Natural } \\
\text { Frequency }(\mathrm{Hz})\end{array}$ & $\begin{array}{c}\text { Power } \\
\text { Output }(\mu \mathrm{W})\end{array}$ & $\begin{array}{c}\text { Normalised Power } \\
\text { Density }\left(\mu \mathrm{W} / \mathrm{cm}^{3} /(\mathrm{m} / \mathrm{s})^{2}\right)\end{array}$ \\
\hline MC70 & 5 & 0.27 & 210 & 22.5 & 562 \\
\hline MC60 & 5 & 0.27 & 196 & 21.4 & 550 \\
\hline MC50 & 5 & 0.29 & 193 & 18.9 & 467 \\
\hline MC40 & 5 & 0.3 & 189 & 17.8 & 396 \\
\hline Elfrink [27] & 4 & 0.2 & 598 & 7.2 & 428 \\
\hline Yu [28] & 51.47 & 0.5 & 234.5 & 66.75 & 519 \\
\hline
\end{tabular}

In order to verify the theory that nonlinear air damping can serve as a soft mechanical stopper and prevent the devices from fracturing when subjected to shocks or large dynamic loading, the air pressure inside the chamber is increased from $1 \mathrm{mbar}, 100 \mathrm{mbar}, 500 \mathrm{mbar}, 800 \mathrm{mbar}$ to 1000 mbar to identify the effect of nonlinear damping on the maximum deflection distance of the cantilever under different air pressure levels.

(a)

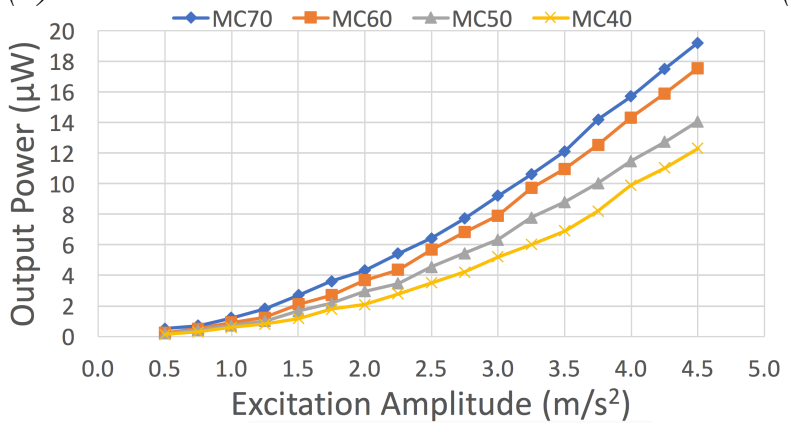

(c)

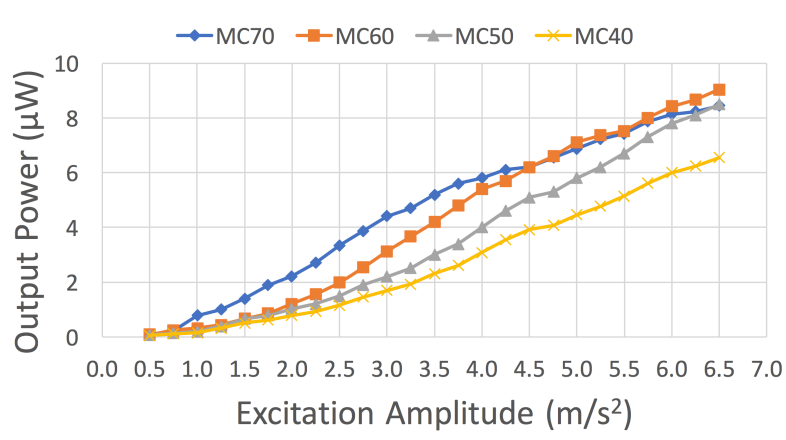

(e)

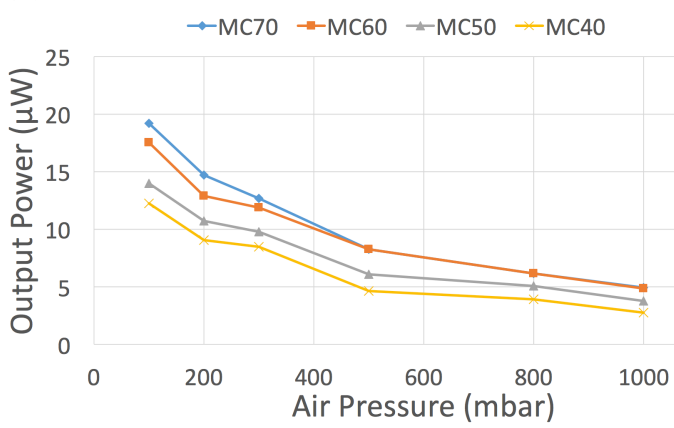

(b)

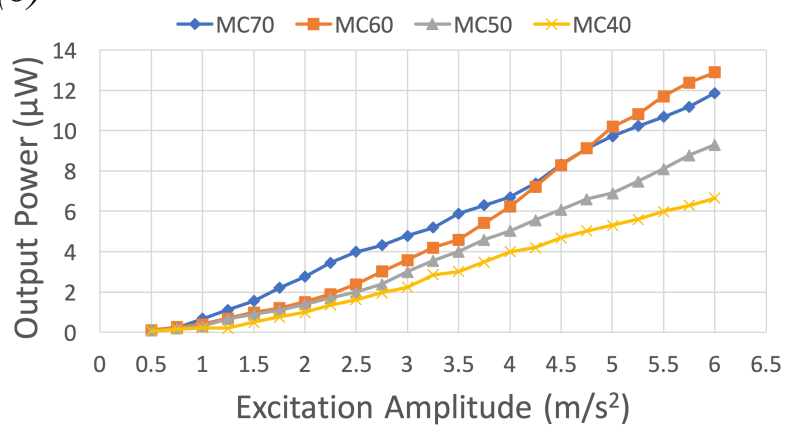

(d)

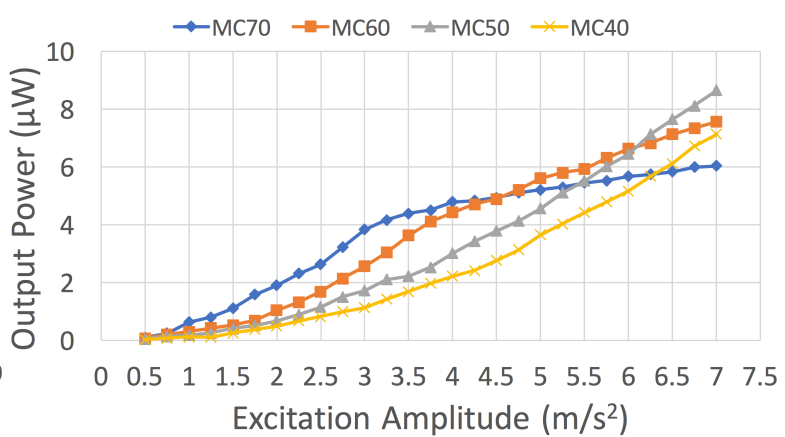

$(f)$

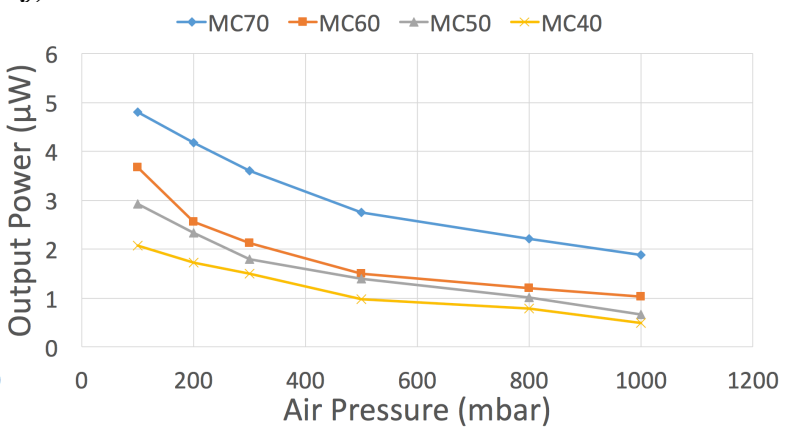

Figure 8. (a) (f) Power curve comparison for $P=$ (a) $100 \mathrm{mbar}$, (b) $500 \mathrm{mbar}$, (c) $800 \mathrm{mbar}$, (d) $1000 \mathrm{mbar}$, (e) Power output as a function of air pressure when excitation is at $4.5 \mathrm{~m} / \mathrm{s}^{2}$ (f) Power output as a function of air pressure when excitation is at $2 \mathrm{~m} / \mathrm{s}^{2}$ 
Fig. 8 (a) depicts the power response of the four devices when the ambient air pressure is 100 mbar. Compared to the power response when the ambient pressure level is 1 mbar, the maximum power output of the PVEH is decreased as the air pressure increases, since the applied energy is dissipated through nonlinear damping by a combination of air drag and squeeze film damping. The drop in maximum power output of the PVEH hence the maximum deflection of the cantilever when the air pressure increases verify the hypothesis that nonlinear air damping can bound the deflection distance of the PVEH.

As seen from the graph, for low air pressure level, the relationship between maximum power output and the excitation amplitude is similar across all four tested devices, as the effect of nonlinear damping is less prominent under lower air levels. The linear relationship of the deflection distance of the devices and excitation amplitude holds true for the dynamic range tested under low nonlinear damping scenarios, which correlate well with the analytical model. Also, the relationship between power output of the devices and the external excitation amplitude is quadratic, by relating the linear relationship between strain and generated voltage of the piezoelectric material in the PVEH and the quadratic relationship between the generated voltage and power output.

Fig. 8 (b), (c) \& (d) depicts the power response of the four devices when the ambient air pressure is 500 mbar, 800 mbar and 1000 mbar. Compared to the power response in Fig. 9 (a), the maximum power output of the PVEH is further decreased as the air pressure increases, which is caused by the diminishing displacement of the PVEH due to increasing nonlinear damping.

Moreover, the maximum power output of the PVEH starts to saturate as the excitation amplitude grows, where the gradient between power output and excitation amplitude decreases as excitation amplitude increases. The amplitude saturation is more prominent for devices with larger proof masses, since nonlinear air damping coefficient is proportional to the projection area of the proof mass and the onset of power saturation starts at lower excitation amplitude as the ambient air pressure increases. As seen from the graph, the power curve for MC70 plateaus around $5 \mathrm{~m} / \mathrm{s}^{2}, 4 \mathrm{~m} / \mathrm{s}^{2}$ and $3 \mathrm{~m} / \mathrm{s}^{2}$ under air pressure of $500 \mathrm{mbar} 800 \mathrm{mbar}$ and $1000 \mathrm{mbar}$ respectively, while for MC40 the power saturation phenomena is not observed even when the ambient air pressure is at 1000 mbar.

The power output is plotted as a function of the ambient air pressure for all four devices when excited at $4.5 \mathrm{~m} / \mathrm{s}^{2}$ and $2 \mathrm{~m} / \mathrm{s}^{2}$ respectively. As seen from Fig. 8 (e), the devices are excited at higher excitation amplitude, although devices with larger proof masses provide higher power output, amplitude saturation is more severe in higher air pressure, as the power output for MC70 is similar to the power output of MC60 when is air pressure is higher than 500 mbar. For Fig. 8 (f), amplitude saturation is less severe when the devices are driven at lower excitation amplitude, thus the power output of MC70 is higher than all the other devices in the entire tested air pressure range.

\subsection{Parameter Extraction}

The relationship between the power curves under different air pressure levels and the nonlinear air damping coefficient in the established Duffing equation with nonlinear damping can be established by nonlinear curve fitting with Levenberg-Marquardt algorithm, which is an iterative technique that locates the minimum of a multivariate function that is expressed as the 
sum of squares of nonlinear real-valued functions [29]. Fig. 9 depicts the values of the nonlinear damping coefficient for MC70 with respect to varying air pressures. Experimental power curves under each air pressure level are obtained and fitted with the theoretical nonlinear Duffing model to obtain the relationship between air pressure and nonlinear damping coefficient [30]. The linear parameters of the cantilever in this study are measured and reported as $\bar{\zeta}=8.16 \times 10^{-4}, \overline{\omega_{n}}=1237 \mathrm{rad} / \mathrm{s}$, and the nonlinear duffing stiffness $\mu$ is derived as $\mu=$ $3 \times 10^{-4}(\mathrm{~ms})^{-2}$ with the experimental result where air pressure is $1 \mathrm{mbar}$ using the LevenbergMarquardt algorithm, where the effect of nonlinear damping in rarefied regime is considered negligible [31]. The piezoelectric coupling coefficient $\theta_{p}=0.25$ for the piezoelectric material being AlN (Aluminum Nitride), the capacitance of the MEMS harvester $C_{p}=0.08 \mathrm{nF}$, and the resistance of the MEMS harvester is $300 \mathrm{k} \Omega$. To calculate the output power from the theoreticl model, the relationship between the cantilever deflection distance of the MEMS vibration energy harvester and the output voltage of the harvester in the theoretical nonlinear Duffing model is obtained by calibrating the deflection distance and the output voltage with a Laser Doppler Vibrometry (Ploytec MSA-500) where air pressure is $1 \mathrm{mbar}$. The output power of the theoretical model is then obtained as $P=\frac{V^{2}}{R}$, where the resistance $R$ of the MEMS vibration energy harvesters of $300 \mathrm{k} \Omega$. Fig. 10 further illustrates the nonlinear damping coefficient for all 4 devices with ambient air pressure ranging from 1 mbar to 1000 mbar.

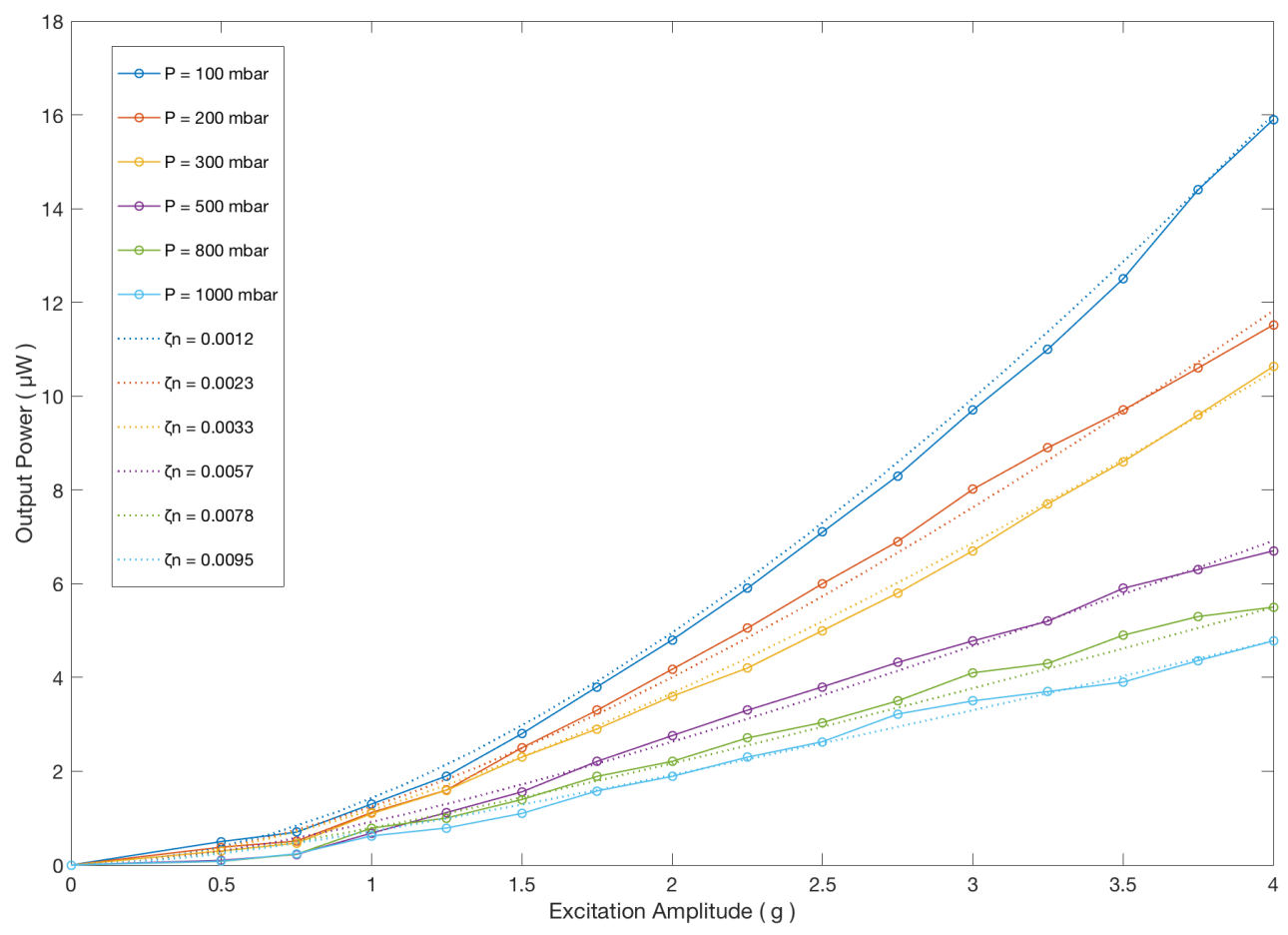

Figure 9. Comparison between the experimental value of power curve for $M C 70$ with varying air pressure with theoretical model to obtain the relationship between nonlinear air damping coefficient with pressure for MC70 


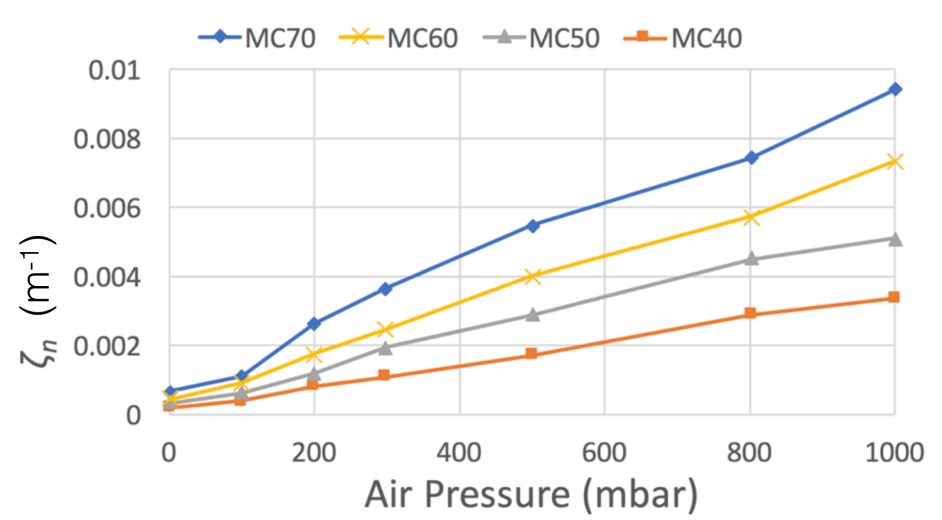

Fig 10. Relationship between nonlinear damping coefficient and air pressure for all four tested devices

\subsection{Dimensionless nonlinear damping coefficient}

Fig.10 demonstrates the relationship between nonlinear damping coefficient and air pressure levels for MC70 by fitting the excitation-power output response of the cantilever using the Levenberg-Marquardt algorithm. As seen from the figure, the nonlinear damping coefficient increases as the ambient pressure increases, which in turn reduces the maximum displacement of the PVEH for the same excitation level conditions.

Moreover, as seen in Fig.10, the nonlinear damping coefficient increases as air pressure increases for all four devices. As the proof mass projection area of the cantilever increases from MC40 to MC70, the nonlinear damping coefficient increases as well, and devices with larger proof masses experience more air drag and squeeze film damping when subjected to external excitation under the same air pressure. This is due to the larger projection areas and more air trapped underneath the film-like space between the cantilever and the bottom-sealed deep cavity chip carrier for devices with larger proof masses.

As shown in the modelling chapter, the relationship between nonlinear damping due to air drag and squeeze film effect has been identified to be a function of the projection area of the proof mass, therefore a dimensionless nonlinear damping coefficient can be defined by normalising the nonlinear damping coefficient for different devices with respect to their specific dimensions. Fig.11 depicts the relationship between the area-normalised nonlinear damping coefficient of each devices under varying air pressure, which shows a close coherent trend between air pressure levels and the dimensionless nonlinear air damping coefficient across all devices. Table 3 records the relative standard deviation value for the dimensionless nonlinear air damping coefficient for all four tested devices for air pressures ranging from 1 mbar to 1000 mbar as a verification that the dimensionless nonlinear damping coefficient can be utilised in modelling the dynamic behaviour of rectangular cantilever with proof mass with nonlinear air damping, and predict the maximum deflection and power output of the MEMS PVEH with varying ambient air pressure levels. 


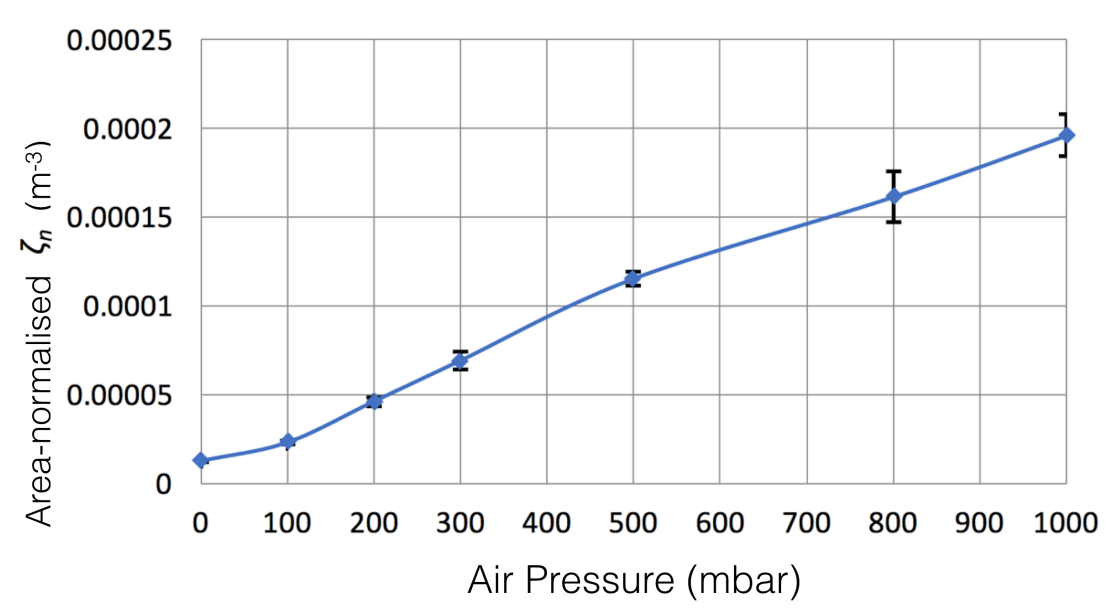

Figure 11. Relationship between area-normalised nonlinear air damping coefficient and air pressure, the error bar depicts the standard deviation among all four tested devices.

Table 3. Relative standard deviation value for dimensionless nonlinear damping coefficient from $P=1$ mbar to $P=1000$ mbar.

\begin{tabular}{|c|c|c|c|c|c|c|c|}
\hline Air Pressure & $\begin{array}{c}1 \\
\text { mbar }\end{array}$ & $\begin{array}{c}100 \\
\text { mbar }\end{array}$ & $\begin{array}{c}200 \\
\text { mbar }\end{array}$ & $\begin{array}{c}300 \\
\text { mbar }\end{array}$ & $\begin{array}{c}500 \\
\text { mbar }\end{array}$ & $\begin{array}{c}800 \\
\text { mbar }\end{array}$ & $\begin{array}{c}1000 \\
\text { mbar }\end{array}$ \\
\hline $\begin{array}{c}\text { Relative Standard } \\
\text { Deviation (\%) }\end{array}$ & 6.73 & 4.57 & 5.52 & 7.28 & 3.18 & 8.93 & 6.02 \\
\hline
\end{tabular}

\section{Design Guidelines}

This section outlines the design considerations for utilising nonlinear air damping as a soft mechanical stopper. The type of loads which the MEMS PVEHs might encounter in typical deployment environments, the topology of the MEMS PVEHs and the corresponding optimal air pressure level is investigated. The design consideration aims to provide a robust design guideline for reliability enhancement of MEMS PVEHs when deployed in excessive dynamic loading and shock loading scenarios.

Srikar and Senturia [32] have performed a series of analysis regarding the dynamic reliability of MEMS structures in shock loading scenarios. Shock loads can be modelled as pulses applied to the MEMS structures with finite duration, where in laboratory settings it is convenient to simulate shock loads with irregular pulse shapes and jagged spectral characteristics as a combination of harmonic shocks pulses using the half-sine waveform [33], where such shock loads can be clearly specified by the duration and maximum acceleration for the purpose of analysing the shock response of the MEMS structures.

Dynamic loadings can be further classified by the duration of the shock $\tau$ relative to the resonant period $T$ and the acoustic transit time $\tau_{a}$ of the MEMS structure, where the shock load is categorised as (a) quasi-static, when the loading duration is much longer than the resonant period, (b) resonant, when the duration of the load is the same order of magnitude of the resonant period of the device and (c) impulse, when the loading duration is much shorter than the resonant period of the device. For MEMS resonators with silicon substrate, the typical acoustic transit time $\tau_{a}$ is less than $0.1 \mu \mathrm{s}$, as the length of the structure being less than $1 \mathrm{~mm}$ and wave propagation speed $\mathrm{c}$ in silicon $=8000 \mathrm{~m} / \mathrm{s}$. The resonant period $T$ for MEMS devices ranges from $10000 \mu \mathrm{s}$ for cavitation sensor to $10 \mu \mathrm{s}$ for accelerometers [32]. 
For vibration energy harvesting applications, the typical acoustic transit time $\tau_{a}$ is less than $0.1 \mu \mathrm{s}$ as mentioned above, and the resonant period $T$ of MEMS PVEHs is designed to be in the order of $1000 \mu$ s to be in the range of the vibration frequency spectrum of the deployed environment, with aim to maximise the response and the power output of the PVEH. Therefore, the vibration frequency of dynamic shocks loads for MEMS PVEHs are usually less than an order of magnitude larger in terms of frequency, making shock loading for MEMS PVEHs fall into the resonant type category.

\subsection{Fracture strength for dynamic loading conditions}

Fracture occurs in MEMS devices when stresses in the structures exceed a certain critical value while subjecting to loading conditions. Ayre et al. [34] have found out that the dynamic fracture strength and the corresponding acceleration experienced by the MEMS devices are dependent on the rate of loading compared to the acoustic transit time $\tau_{a}$ of the MEMS structure, where stress wave induced fracture is only significant when the loading duration is on the same order of magnitude of the acoustic transit time. For MEMS resonators, the typical acoustic transit time $\tau_{a}$ is less than $0.1 \mu \mathrm{s}$, which is several orders of magnitude lesser than the loading duration of the excitation. In this case, stress wave induced fracture should be negligible and the fracture strength of MEMS devices under dynamic excitation should be taken the same as the static fracture strength of their corresponding composing materials. Moreover, according the Brockenbrough et al. [35], the dynamic fracture strength is usually greater than the corresponding static fracture strength of brittle materials by a factor between 1.0 to 1.7, therefore the use of static fracture strength in calculating the maximum allowable deflection distance for MEMS PVEHs when subjected to resonant type excitation is conservative and reasonable.

As a result, the critical stress of MEMS PVEHs when subjected to resonant type dynamic loadings should be treated as the same as the static fracture strength, given the frequencies and the duration of the shocks MEMS PVEHs experience are on the same order of magnitude as the resonant period $T$ of the MEMS devices. The failure criteria for MEMS PVEHs under resonant type dynamic loading conditions can therefore be defined according to the Weibull probability distribution function deduced by Greek et al. [36], with the probability of fracture (P) of silicon as:

$$
P=1-e^{\left.\left(-6 \times 10^{-9}\left(\frac{\sigma}{102}\right)\right)^{11}\right)}
$$

where $\sigma$ is the stress experienced by the MEMS PVEH during resonant type loading (in MPa). For designing a robust MEMS PVEH, the probability of fracture $P$ is set to 0.2 as a threshold to provide sufficient confidence that the nonlinear air damping can lower the stress experienced by the MEMS PVEH during resonant type excitations to avoid fracture.

For a MEMS PVEH with a rectangular cantilever topology, the maximum bending stress when a distributed load is applied occurs at the anchor of the cantilever. If a resonant type dynamic load is applied to the MEMS PVEH with length $l$, the resulting maximum stress in the PVEH is a function of the maximum deflection of the device, which can be obtained as [37]: 


$$
\sigma_{\max }=\frac{E I}{Z} \delta \ddot{(0)}=\frac{3 E I}{Z} \frac{\delta_{l}}{l^{2}}
$$

where $E, I$ and $Z$ are the Young's Modulus, moment of inertia and the section modulus of the $\mathrm{PVEH}$, respectively and $\delta_{l}$ denotes the maximum deflection of the device under resonant type loading.

Since the maximum deflection of the device under the same excitation amplitude can be altered by changing the air pressure of the package for MEMS PVEH, the maximum stress the device experiences during resonant type loading can also be reduced by increasing the air pressure inside the MEMS PVEH package.

For a given environment, two parameters, namely excitation range and failure limit, can be defined to properly determine the air pressure level inside the MEMS PVEH package to optimize with respect to the trade-off between low fracture probability and highest power output. The failure limit for a given device is defined as the maximum deflection which corresponds to the fracture strength calculated by the fracture probability equation. As stated above, for robust design, the fracture probability is set to be 0.2 to provide a guideline such that the device would survive the stress related to the dynamic loading from the environment. The excitation range is the maximum excitation amplitude the device can withstand before reaching the failure limit. By utilising nonlinear air damping as soft mechanical stopper, the excitation range can be extended by increasing the air pressure in the MEMS PVEH package, meaning the PVEH is able to be employed into environment with higher dynamic loading scenarios without fracturing.

Fig. 12 is the layout of the design flowchart when selecting the appropriate pressure for a given MEMS PVEH by taking into account of the dimensions and the deployment conditions of the MEMS PVEH. The maximum allowable strength can be calculated using Eqn.18 and setting the probability of fracture to a desirable value. The maximum stress experienced by the MEMS PVEH can be reduced by the employment of nonlinear air damping as nonlinear damping limits the maximum allowable deflection distance of the device. The appropriate air pressure level can be found by identifying the maximum excitation amplitude in the deployment environment, and the relationship between air pressure and nonlinear air damping coefficient, considering the geometry of the device and the gap between the device and the substrate. 
Calculate fracture strength for

the MEMS PVEH under dynamic

load based on Weibull

probability density function

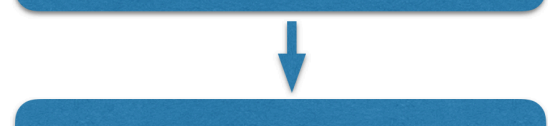

Calculate maximum allowable

deflection distance for MEMS

PVEH based on fracture strength
Identify maximum excitation amplitude from the environment

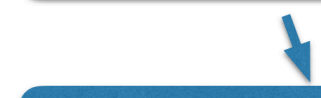

Calculate nonlinear air damping coefficient from deflection distance and maximum external excitation with nonlinear fitting method

1

Calculate appropriate air pressure level for performance and

reliability optimisation

Figure 12. Design guideline for calculating the optimal air pressure for MEMS PVEH as soft mechanical stoppers for performance and reliability optimisation

\section{Nonlinear air damping application}

\subsection{Effect of gap size on nonlinear damping}

As mentioned in the modelling chapter, nonlinear air damping originates from a combination of air drag and squeeze film damping. It is also shown that the effect of squeeze film damping is enhanced when the gap between the vibrating cantilever and the bottom substrate is decreased. Fig. 13 therefore compares the power curve of MC70 operating in 300 mbar with three different gaps between the cantilever and the bottom chip carrier to demonstrate the effect of gap size on nonlinear damping and the dynamic behaviour of the system. The depth of the chip carriers is chosen to be $3 \mathrm{~mm}, 2 \mathrm{~mm}$ and a hollow bottom, as shown in Fig. 14 .

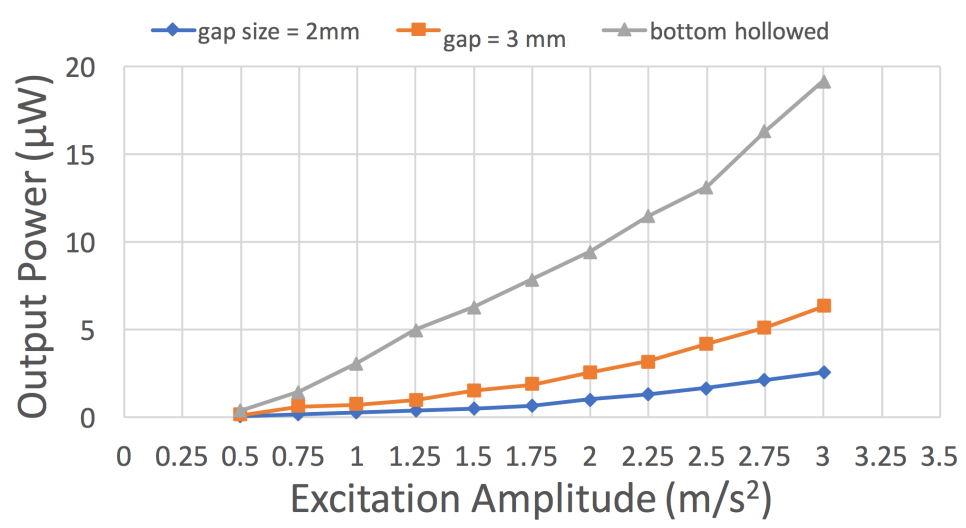

Figure 13. Power curve for MC70 operating at $p=300$ mbar with different gap sizes 


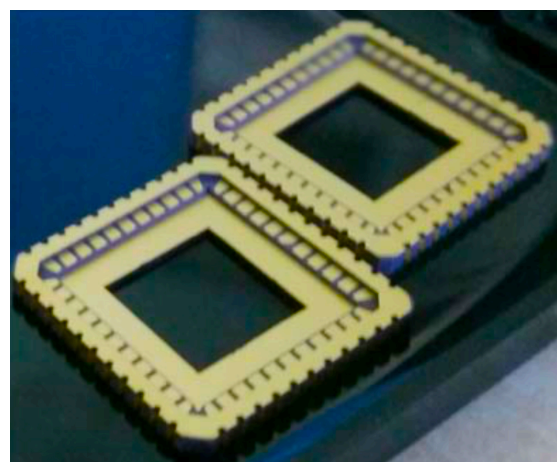

Figure 14. Photograph of chip carriers with through holes at the bottom to demonstrate the effect of gap size on nonlinear air damping

As seen from the graph, the gap size between the cantilever and the bottom of the chip carrier has a dramatic effect on the power output of the PVEH, as the peak power for the hollowed, 3 $\mathrm{mm}$ and $2 \mathrm{~mm}$ gap size being $19.2 \mu \mathrm{W}, 6.33 \mu \mathrm{W}$ and $2.55 \mu \mathrm{W}$, respectively. The nonlinear damping coefficient in these three cases can be obtained by utilising the Levenberg-Marquardt algorithm described in the parameter extraction paragraph, and the values of the fitted nonlinear damping coefficients along with the peak power output are recorded in Table 4 . The nonlinear damping mechanism in the hollowed bottom case can be approximated as being only originating from air drag, since the gap size can be seen as infinitely large thus eliminating the effect of squeeze film damping. The nonlinear damping coefficient caused by squeeze film damping can be obtained for both $3 \mathrm{~mm}$ and $2 \mathrm{~mm}$ gap size by comparing to the value for the hollowed bottom. It can be seen that the nonlinear damping coefficient is 0.0062 and 0.0009 for squeeze film damping when the gap size is $3 \mathrm{~mm}$ and $2 \mathrm{~mm}$, respectively. The ratio between these two coefficients is 7.12 , which is within $10 \%$ of the predicted value from the theoretical model where the nonlinear damping coefficient caused by squeeze film damping is inversely proportional to the cubic value of the gap size as:

$$
\xi_{2 n} \propto \frac{1}{g_{0}{ }^{3}}
$$

Table 4. Nonlinear damping coefficient for different gap sizes

\begin{tabular}{|c|c|c|}
\hline Gap Size & Peak Power Output $(\mu \mathrm{W})$ & $\zeta_{\mathrm{n}} \mathrm{N} /\left(\mathrm{m} / \mathrm{s}^{2}\right)$ \\
\hline Hollowed & 19.2 & 0.00083 \\
\hline $3 \mathrm{~mm}$ & 6.33 & 0.0017 \\
\hline $2 \mathrm{~mm}$ & 2.55 & 0.007 \\
\hline
\end{tabular}

\subsection{Noise excitation characterisation}

Finally, random noise excitation experiments are performed to test the idea of utilising nonlinear air damping as a soft stopper for MEMS PVEHs operating in real-world scenarios. The input vibration for the system is a band-limited white noise excitation ( $10 \mathrm{~Hz}$ to $2 \mathrm{kHz}$ ), and Fig.16 shows the response and the power output of the MEMS PVEH under different air pressure levels when subjected to $7 \mathrm{~m} / \mathrm{s}^{2}$ of band-limited white noise $\left(0.025 \mathrm{~g}^{2} \mathrm{~Hz}^{-1}\right)$. 
Fig. 15 (a) illustrates the time response and RMS voltage output of MC70 when subjected to 7 $\mathrm{m} / \mathrm{s}^{2}$ of band-limited white noise $\left(0.025 \mathrm{~g}^{2} \mathrm{~Hz}^{-1}\right)$ when the air pressure is at $10 \mathrm{mbar}, 300 \mathrm{mbar}$ and 800 mbar respectively and Fig.16 (b) further depicts the power output as a function of air pressure under random excitation, as seen from the graph, as the air pressure increases, the maximum deflection of the MEMS PVEH and the power output decrease, thus demonstrating the utilisation of nonlinear air damping as a soft stopper when deployed in random excitation environments.

(a)

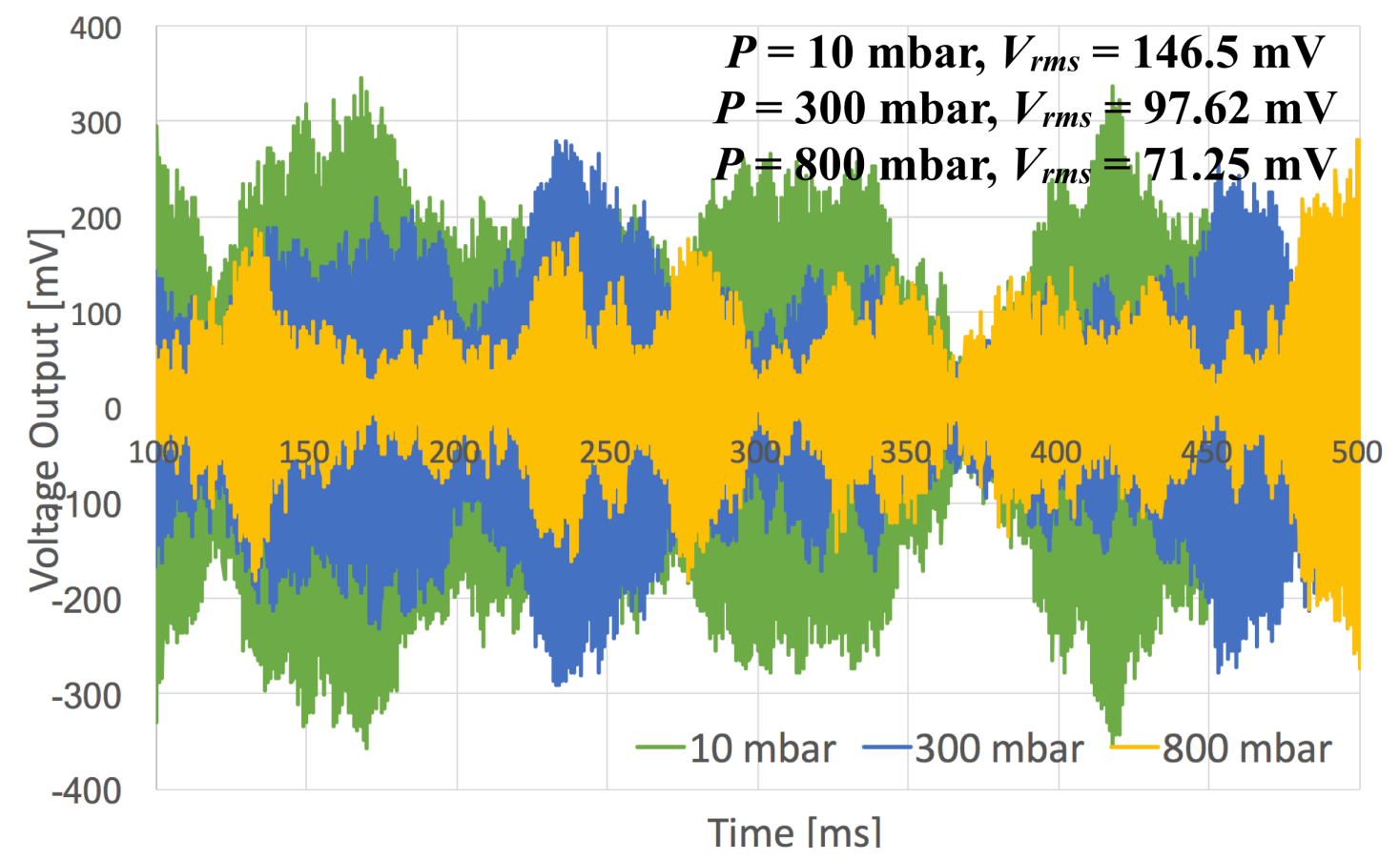

(b)

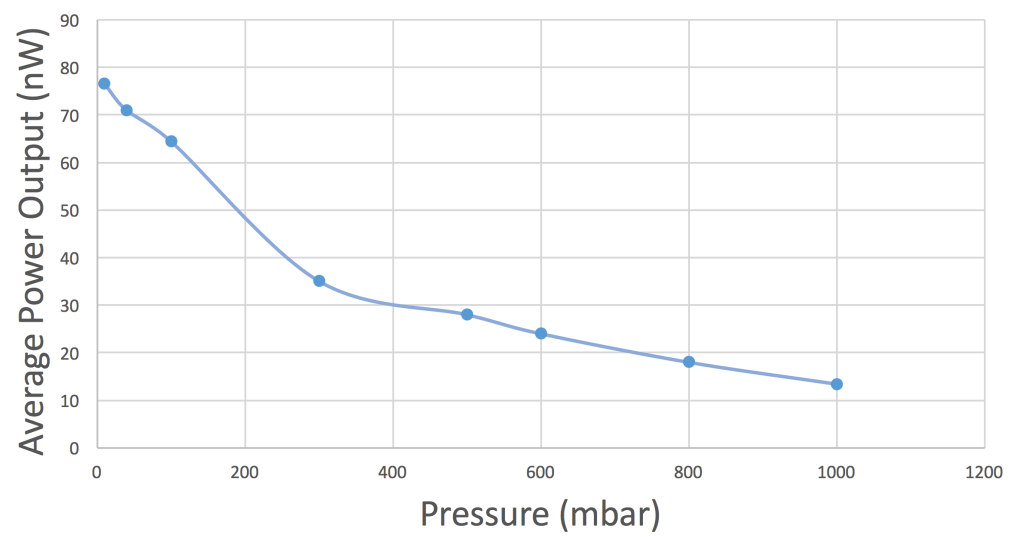

Figure 15(a) \& (b). (a) Random excitation response of MC70 with $1000 \mathrm{~Hz}$ band-limited white noise. (b)Average power output as a function of the air pressure for MC70 


\section{Conclusion and Future work}

While vacuum packaging is typically used in MEMS applications to minimise air damping and increase the quality factor and performance of the MEMS devices, this study demonstrates the utilisation of nonlinear air damping as soft stopper for MEMS PVEH to increase its robustness for shock and excessive dynamic loading.

The theoretical model for nonlinear damping is constructed based on Duffing equation with nonlinear damping and experimentally verified, whereas nonlinear damping mechanisms for cantilever vibrating in air have been identified to be a combination of both air drag and squeeze film damping.

The study shows that the power curve of the PVEH can be altered by varying the air pressure level, the gap size between the PVEH and the substrate, and the projection area of the PVEH. Area-normalised nonlinear damping coefficient were obtained to demonstrate the relationship between the projection area of the MEMS PVEH and the nonlinear damping forces under different air pressure levels.

The failure limit and excitation range of a rectangular MEMS PVEH with nonlinear air damping subjected to external excitation is demonstrated, and design guidelines for utilising nonlinear air damping as soft mechanical stopper has been constructed for rectangular MEMS PVEH where optimal air pressure can be calculated when knowing the fracture strength of the device and the relationship between pressure and nonlinear damping coefficient for the particular device. 


\section{Reference:}

[1] M. Esashi, "Wafer level packaging of MEMS," Journal of Micromechanics and Microengineering, vol. 18, p. 073001, 2008.

[2] J. Wu, G. K. Fedder, and L. R. Carley, "A low-noise low-offset capacitive sensing amplifier for a 50-/spl mu/g//spl radic/Hz monolithic CMOS MEMS accelerometer," IEEE Journal of Solid-State Circuits, vol. 39, pp. 722-730, 2004.

[3] A. Ajayaghosh, V. K. Praveen, and C. Vijayakumar, "Organogels as scaffolds for excitation energy transfer and light harvesting," Chemical Society Reviews, vol. 37, pp. 109-122, 2008.

[4] A. Cuadras, M. Gasulla, and V. Ferrari, "Thermal energy harvesting through pyroelectricity," Sensors and Actuators A: Physical, vol. 158, pp. 132-139, 2010.

[5] A. Erturk, J. Hoffmann, and D. Inman, "A piezomagnetoelastic structure for broadband vibration energy harvesting," Applied Physics Letters, vol. 94, p. 254102, 2009.

[6] Y. Jia and A. A. Seshia, "Power optimization by mass tuning for MEMS piezoelectric cantilever vibration energy harvesting," Journal of Microelectromechanical Systems, vol. 25, pp. 108-117, 2016.

[7] M. Renaud, Z. Wang, M. Jambunathan, S. Matova, R. Elfrink, M. Rovers, et al., "Improved mechanical reliability of MEMS piezoelectric vibration energy harvesters for automotive applications," in Micro Electro Mechanical Systems (MEMS), 2014 IEEE 27th International Conference on, 2014, pp. 568-571.

[8] M. Renaud, T. Fujita, M. Goedbloed, C. de Nooijer, and R. van Schaijk, "Shock reliability analysis and improvement of MEMS electret-based vibration energy harvesters," Journal of Micromechanics and Microengineering, vol. 25, p. 104010, 2015.

[9] S.-T. Chen, S. Du, E. Arroyo, Y. Jia, and A. Seshia, "Utilising Nonlinear Air Damping as a Soft Mechanical Stopper for MEMS Vibration Energy Harvesting," in Journal of Physics: Conference Series, 2016, p. 012098.

[10] T. J. Kaźmierski and S. Beeby, Energy harvesting systems: principles, modeling and applications: Springer Science \& Business Media, 2010.

[11] C. Williams and R. B. Yates, "Analysis of a micro-electric generator for microsystems," sensors and actuators A: Physical, vol. 52, pp. 8-11, 1996.

[12] D. Guyomar, A. Badel, E. Lefeuvre, and C. Richard, "Toward energy harvesting using active materials and conversion improvement by nonlinear processing," IEEE transactions on ultrasonics, ferroelectrics, and frequency control, vol. 52, pp. 584-595, 2005.

[13] A. Triplett and D. D. Quinn, "The effect of non-linear piezoelectric coupling on vibration-based energy harvesting," Journal of Intelligent Material Systems and Structures, vol. 20, pp. 1959-1967, 2009.

[14] P. R. Dahl, "Solid friction damping of mechanical vibrations," AIAA journal, vol. 14, pp. 1675-1682, 1976.

[15] W. E. Baker, W. E. Woolam, and D. Young, "Air and internal damping of thin cantilever beams," International Journal of Mechanical Sciences, vol. 9, pp. 743-766, 1967.

[16] S. Roundy, P. K. Wright, and J. M. Rabaey, Energy scavenging for wireless sensor networks: with special focus on vibrations: Springer Science \& Business Media, 2012.

[17] F. Lu, H. Lee, and S. Lim, "Modeling and analysis of micro piezoelectric power generators for micro-electromechanical-systems applications," Smart Materials and Structures, vol. 13, p. 57, 2003. 
[18] S.-N. Chen, G.-J. Wang, and M.-C. Chien, "Analytical modeling of piezoelectric vibration-induced micro power generator," Mechatronics, vol. 16, pp. 379-387, 2006.

[19] A. Erturk and D. J. Inman, "Issues in mathematical modeling of piezoelectric energy harvesters," Smart Materials and Structures, vol. 17, p. 065016, 2008.

[20] A. Erturk and D. J. Inman, "A distributed parameter electromechanical model for cantilevered piezoelectric energy harvesters," Journal of vibration and acoustics, vol. 130, p. 041002, 2008.

[21] T. Tan, Z. Yan, and M. Hajj, "Electromechanical decoupled model for cantilever-beam piezoelectric energy harvesters," Applied Physics Letters, vol. 109, p. 101908, 2016.

[22] B. Ravindra and A. K. Mallik, "Role of Nonlinear Dissipation in Soft Duffing Oscillators," Physical Review E, vol. 49, pp. 4950-4954, Jun 1994.

[23] S. F. Hoerner, Fluid-dynamic drag: practical information on aerodynamic drag and hydrodynamic resistance: Hoerner Fluid Dynamics, 1965.

[24] H. Hosaka, K. Itao, and S. Kuroda, "Damping characteristics of beam-shaped microoscillators," Sensors and Actuators A: Physical, vol. 49, pp. 87-95, 1995.

[25] W. Choi, Y. Jeon, J.-H. Jeong, R. Sood, and S.-G. Kim, "Energy harvesting MEMS device based on thin film piezoelectric cantilevers," Journal of Electroceramics, vol. 17, pp. 543-548, 2006.

[26] H. Li, C. Tian, and Z. D. Deng, "Energy harvesting from low frequency applications using piezoelectric materials," Applied physics reviews, vol. 1, p. 041301, 2014.

[27] R. Elfrink, M. Renaud, T. Kamel, C. De Nooijer, M. Jambunathan, M. Goedbloed, et al., "Vacuum-packaged piezoelectric vibration energy harvesters: damping contributions and autonomy for a wireless sensor system," Journal of Micromechanics and Microengineering, vol. 20, p. 104001, 2010.

[28] H. Yu, J. Zhou, L. Deng, and Z. Wen, "A vibration-based MEMS piezoelectric energy harvester and power conditioning circuit," Sensors, vol. 14, pp. 3323-3341, 2014.

[29] J. J. Moré, "The Levenberg-Marquardt algorithm: implementation and theory," in Numerical analysis, ed: Springer, 1978, pp. 105-116.

[30] M. I. Lourakis, "A brief description of the Levenberg-Marquardt algorithm implemented by levmar," Foundation of Research and Technology, vol. 4, pp. 1-6, 2005.

[31] H. Sumali, "Squeeze-film damping in the free molecular regime: model validation and measurement on a MEMS," Journal of Micromechanics and Microengineering, vol. 17, p. 2231, 2007.

[32] V. Srikar and S. D. Senturia, "The reliability of microelectromechanical systems (MEMS) in shock environments," Journal of Microelectromechanical Systems, vol. 11, pp. 206-214, 2002.

[33] T. G. Brown, B. Davis, D. Hepner, J. Faust, C. Myers, C. Muller, et al., "Strap-down microelectromechanical (MEMS) sensors for high-g munition applications," IEEE Transactions on Magnetics, vol. 37, pp. 336-342, 2001.

[34] R. Ayre, "Nonlinear dynamic response of elastic slider-crank mechanism," Journal of Engineering for Industry, p. 251, 1971.

[35] J. Brockenbrough, S. Suresh, and J. Duffy, "An analysis of dynamic fracture in microcracking brittle solids," Philosophical Magazine A, vol. 58, pp. 619-634, 1988.

[36] S. Greek, F. Ericson, S. Johansson, M. Fürtsch, and A. Rump, "Mechanical characterization of thick polysilicon films: Young's modulus and fracture strength evaluated with microstructures," Journal of Micromechanics and Microengineering, vol. 9, p. 245, 1999. 
[37] E. Suhir, "Is the maximum acceleration an adequate criterion of the dynamic strength of a structural element in an electronic product?," IEEE Transactions on Components, Packaging, and Manufacturing Technology: Part A, vol. 20, pp. 513-517, 1997. 